Reprod. Nutr. Dévelop., 1986, 26 (5 A), 1137-1149.

\title{
Influence de la caecotrophie sur la production, l'absorption et l'utilisation des acides organiques chez le lapin
}

\author{
Michèle VERNAY
}

Laboratoire de Physiologie de la Digestion et de la Nutrition Université Paul Sabatier,

2 , rue F. Magendie, 31400 Toulouse, France.

Summary. Effect of caecotrophy on the production, absorption and utilization of organic acids in the rabbit.

The influence of caecotrophy on organic acid production and behaviour was studied for 4 days in rabbits with and without a collar preventing caecotrophy. Volatile fatty acids (VFA) and lactate were measured in the digestive material and the corresponding venous blood. Blood was removed from the gastric, intestinal, caecocolonic, portal and hepatic veins and the inferior vena cava of anaesthetized rabbits. Arterial blood was also collected.

A comparison of the data obtained from rabbits with and without a collar showed significant differences. When caecotrophy was prevented, lactic fermentation in the stomach ceased and the lactate level dropped in venous and arterial blood. Meanwhile, VFA production in the foregut (stomach and intestine) stopped, whereas it augmented in the hindgut ; VFA enrichment of the caecocolonic and portal blood was greater when the rabbits were subjected to a stercoral fast. The liver removed about $15 \%$ of the lactate reaching it, while acetate, propionate and butyrate uptakes were 21,59 and $76 \%$, respectively, in rabbits with caecotrophy. The corresponding values for those without caecotrophy were : $20 \%$ lactate, $43 \%$ acetate, $70 \%$ propionate and $84 \%$ butyrate. The plasma venous-arterial difference in acetate was negative in the peripheral blood : uptake was about $16 \%$ for the controls and $27 \%$ for rabbits without caecotrophy; the other VFA were not involved. The variations of the levels of VFA in the arterial blood were not significant ; only lactate concentration diminished by about $30 \%$.

Preventing caecotrophy augmented digestive production, and the absorption of VFA in the rabbit hindgut and their metabolism in the liver and extra-hepatic tissues also increased.

\section{Introduction.}

Le lapin est un herbivore adapté à l'ingestion de grandes quantités de végétaux qu'il consomme de façon quasi continue par petites prises (Hörnicke et al., 1976 ; Fioramonti, Bueno et Candau, 1978) ; à cette alimentation traditionnelle, se surajoute l'alimentation stercorale. En effet, une fois par jour, le lapin pratique la caecotrophie ; cela correspond à l'ingestion dans le courant de la matinée d'une 
production fécale spéciale appelée : fèces molles ou caecotrophes. Ces fèces riches en protéines totales (Proto, 1980 ; Vernay, 1985), sont constituées pour moitié environ de corps bactériens et pour l'autre moitié par des résidus alimentaires incomplètement digérés. Cette pratique permet l'ensemencement continuel des parties antérieures du tube digestif : estomac et intestin grêle (Smith, 1965), une meilleure utilisation des protéines et de la matière sèche de la ration (Huang, Ulrich et McCay, 1954 ; Battaglini, 1968 ; Spreadbury, 1978), une supplémentation en vitamines B, C et K (Kulwich, Struglia et Pearson, 1953 ; Megard, 1970) et une récupération des minéraux (Hörnicke et Björnhag, 1980).

Pour le lapin normalement alimenté, la dégradation de la cellulose, de l'amidon et de différents sucres, par les enzymes bactériennes digestives, donne lieu à la formation d'acides gras volatils (acétate, propionate, butyrate) et de lactate (Elsden et al., 1946 ; Griffiths et Davies, 1963 ; Henning et Hird, 1972a).

A notre connaissance peu de travaux concernent I'incidence du jeûne stercoral sur la production et le devenir des acides gras volatils (AGV) et du lactate chez le lapin. Les recherches ont été le plus souvent limitées à l'étude des fluctuations du taux des AGV dans le digesta (Le Bars, Guémon et Demaux, 1971 ; Vernay et Raynaud, 1975). C'est ce qui nous a conduit à rechercher quels pouvaient être les effets de la non-ingestion des caecotrophes sur la teneur en AGV et lactate dans le bol alimentaire ainsi que dans le sang veineux et artériel.

\section{Matériel et méthodes.}

Animaux. - Au total, 50 lapins de race commune, d'un poids moyen de $2,5 \pm 0,1 \mathrm{~kg}$ sont utilisés. Ils sont nourris ad libitum de luzerne et d'avoine et répartis en deux lots :

- un lot de 24 animaux libres de pratiquer la caecotrophie (témoins) ;

- un lot de 26 animaux privés expérimentalement d'alimentation caecotrophique pendant 4 jours. Pour empêcher l'ingestion des fèces molles émises, les lapins sont munis d'un collier plastique de $27 \mathrm{~cm}$ de diamètre et sont placés sur un grillage à mailles suffisamment larges pour laisser passer les caecotrophes (Kametaka, 1967).

Pour les deux groupes d'animaux, nous pesons quotidiennement la nourriture distribuée, restante et gaspillée. En règle générale, les expériences sont réalisées soit le matin entre 6 et 11 heures, lorsque les sujets produisent des fèces molles (FM), soit l'après-midi entre 14 et 16 heures lorsqu'ils exonèrent des fèces dures (FD).

Techniques physiologiques. - Les résultats des travaux de Desbals (1963) et de Bito et Eakins (1969) obtenus chez le lapin nous ont orienté vers le choix d'une anesthésie au pentobarbital. En effet, ces auteurs signalent que ce barbiturique n'entraîne que de très légères modifications dans la biochimie sanguine : la diminution de la pression en oxygène n'est pas significative, la glycémie et la natrémie ne sont pas affectées, seule la kaliémie augmente discrètement. 
Après anesthésie au pentobarbital $(20 \mathrm{mg} / \mathrm{kg})$ et laparotomie, du sang $(0,6 \mathrm{ml})$ est prélevé dans les différentes veines du tube digestif (gastrique, iléale, caecale, colique). Pour l'étude du bilan hépatique des prélèvements sanguins sont réatisés dans la veine hépatique (sang efférent) par ponction directe à l'intérieur du lobe gauche du foie, puis simultanément dans la veine porte et l'aorte abdominale (sang afférent). Une prise de sang est également effectuée dans la veine cave inférieure. Le temps pour chaque prise de sang ne dépasse pas 15 secondes et la veine est immédiatement clampée à la fin de l'opération. Le sang récolté sur héparine est centrifugé ( $2 \mathrm{~min}, 10000 \mathrm{xg}$ ) et le plasma conservé à $-20^{\circ} \mathrm{C}$.

Après sacrifice de l'animal, des échantillons de matériel digestif 10,2 à $1 \mathrm{~g})$ sont placés dans des microtubes Eppendorf et immédiatement congelés à $-20^{\circ} \mathrm{C}$.

Techniques biochimiques. - Les acides gras volatils sont dosés par chromatographie en phase gazeuse sur Intersmat IGC 120 DFL, dans le matériel digestif et dans le plasma selon les techniques préconisées par Rémésy et Demigné (1973, 1974, 1976). La teneur en L-lactate est déterminée par l'action de la L-lactate déshydrogénase (EC.1.1.1.27) de Boehringer en présence de NAD, selon le procédé de Hohorst (1965), mais avec glycine $0,25 \mathrm{M}$, hydrazine $0,20 \mathrm{M}$ et

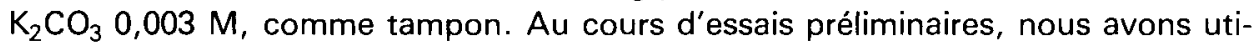
lisé la L-lactate déshydrogénase (EC. 1.1.1.27) et la D-lactate déshydrogénase (EC.1.1.1.28). Contrairement aux résultats de Hörnicke et Mackiewicz (1976), obtenus au cours d'incubation in vitro de fèces molles, nous n'avons pas décelé la présence de D-lactate, aussi bien dans le matériel digestif que dans le plasma. Giesecke, Fabritius et Wallenberg (1981), Stangassinger, Wallenberg et Giesecke (1982) confirment cela et l'expliquent par la « vie " extrêmement fugitive du Dlactate, très rapidement transformé par les bactéries lactolytiques. En conséquence, seul le dosage du L-lactate a été réalisé.

Expression des résultats. Méthodes statistiques. - Chez le polygastrique normalement alimenté, de même que chez le monogastrique, il est montré que le débit hépatique se répartit à raison de $70 \%$ environ pour la veine porte et de $30 \%$ pour l'artère hépatique (Greenway et Stark, 1971; Bergman et Wolff, 1971 ; Rémésy et Demigné, 1982). En conséquence, les concentrations des métabolites dans le plasma afférent sont calculées ainsi : 0,7 (veine porte) $+0,3$ (artère). Le bilan hépatique correspond à la différence entre la veine hépatique et le plasma afférent. Le pourcentage de libération ou de captation est le rapport du bilan hépatique sur le plasma afférent $\times 100$.

Les résultats sont exprimés en $\mathrm{mM}$ pour le matériel digestif et en $\mu \mathrm{mol}$ pour $100 \mathrm{ml}$ de plasma. Pour chaque série expérimentale la moyenne arithmétique est accompagnée de l'erreur standard $(m \pm S E$ ). L'exploitation statistique des résultats est effectuée à l'aide du test de Student sur les valeurs moyennes ou en partant de l'analyse des données par paire. Les différences sont considérées comme significatives quand $P<0.05$. 


\section{Résultats.}

Le port du collier ne modifie pas, de façon significative, l'ingestion d'aliment : $124 \pm 4 \mathrm{~g} / 24 \mathrm{~h}$ pour les témoins et $127 \pm 9 \mathrm{~g} / 24 \mathrm{~h}$ pour les sujets privés expérimentalement de caecotrophie.

L'acidité organique du bol alimentaire est significativement modifiée par un jeune stercoral de 4 jours (tabl. 1). Dans le matériel gastrique, les teneurs en AGV et lactate s'effondrent $(P<0,001)$. Dans les autres compartiments du tube digestif, une distinction doit être faite entre les AGV d'une part et le lactate d'autre part, leur évolution étant différente. Si l'absence d'alimentation caecotrophique n'affecte pas significativement le taux de lactate dans le bol alimentaire qui transite du duodénum au rectum, il n'en est pas de même pour les AGV. En effet, dans I'intestin grêle, l'acidité volatile totale est fortement diminuée $(P<0,001)$, tandis qu'elle est significativement plus élevée dans les régions postérieures du tube digestif : caecum $(P<0,01)$. A l'élévation du taux des $A G V$ correspond une

\section{TABLEAU 1}

Les effets d'un jeâne stercoral de 4 jours sur la teneur (mM) en lactate et acides gras volatils du bol alimentaire.

\begin{tabular}{|c|c|c|c|c|c|c|c|}
\hline \multirow[b]{2}{*}{ Matériel } & \multicolumn{3}{|c|}{ Lactate } & \multicolumn{4}{|c|}{ Acidité volatile totale } \\
\hline & $\begin{array}{c}\text { Témoins } \\
\text { (24) }\end{array}$ & & $\begin{array}{c}\text { Jeûne } \\
\text { (26) }\end{array}$ & $\begin{array}{l}\text { Témoins } \\
\text { (24) }\end{array}$ & & \multicolumn{2}{|c|}{$\begin{array}{c}\text { Jeûne } \\
\text { (26) }\end{array}$} \\
\hline \multirow{2}{*}{\multicolumn{8}{|c|}{ Estomac }} \\
\hline & & & & & & & \\
\hline rumuts & & & & & & & \\
\hline & 0,7 & & $\pm 0,3$ & $\pm 1,9$ & & \pm & 1,3 \\
\hline Corpus & 3,4 & $* *$ & $-1,9$ & 7,8 & $* *$ & & 2,6 \\
\hline & $\pm \quad 0,4$ & & $\pm 0,2$ & 1,3 & & \pm & 0,9 \\
\hline Antrum & 2,4 & * & 1,6 & 4,1 & & & 2,2 \\
\hline & $\pm \quad 0,2$ & & $\pm 0,3$ & $\pm 0,8$ & & \pm & 0,7 \\
\hline \multicolumn{8}{|l|}{ Intestin grêle } \\
\hline Duodénum & 7,2 & & 7,0 & 3,8 & * & & 1,6 \\
\hline Deverium & $\begin{array}{r}7,2 \\
+\quad 0,5\end{array}$ & & $\pm 0,6$ & 0,6 & & \pm & 0,7 \\
\hline Jéjunum & 10,5 & & 8,9 & 4,4 & $* *$ & & 1,8 \\
\hline & $\pm \quad 1,7$ & & $\pm 0,6$ & $\pm \quad 0,7$ & & \pm & 0,6 \\
\hline Iléon & $\quad 9,1$ & & 9,3 & 5,0 & $*$ & & 3,0 \\
\hline & $\pm \quad 1,5$ & & $\pm 0,6$ & $\pm \quad 0,6$ & & \pm & 0,7 \\
\hline \multicolumn{8}{|l|}{ Gros intestin } \\
\hline Caecum & 3,7 & & 3,5 & 73,8 & $* *$ & & 03,3 \\
\hline & $\pm \quad 0,7$ & & $\pm 0,7$ & $\pm \quad 5,9$ & & \pm & 7,4 \\
\hline Côlon 1 & 3,8 & & 3,6 & 48,6 & & & 69,4 \\
\hline & $\pm \quad 13$ & & $+0,9$ & $\begin{array}{r}5,6 \\
\pm \quad 6\end{array}$ & & \pm & 6,2 \\
\hline Côlon 2 & 2,7 & & 2,7 & 32,2 & & & 47,0 \\
\hline & $\begin{array}{r}12 \\
+\quad 12\end{array}$ & & +16 & $\begin{array}{r}8,8 \\
+\quad 8\end{array}$ & & \pm & 9,2 \\
\hline Côlon $_{3}$ & $-2,2$ & & 2,6 & $-30,5$ & & & 37,0 \\
\hline & $\pm 1,6$ & & $\pm 1,8$ & $\pm \quad 8,1$ & & \pm & 9,5 \\
\hline
\end{tabular}

- moyenne $\pm \mathrm{SE}$

- côlon proximal : première (1) et deuxième (2) moitié ; côlon distal (3) :

- ( ) : nombre total d'échantillons ; sur les 24 témoins, 10 produisent des FM et 14 des FD. Pour les 26 animaux au jeûne, 10 exonèrent des FM et 16 des FD.

- Test de Student : comparaison témoins/jeûne stercoral ;

- significations : ${ }^{*} \mathrm{P}<0,05 ;{ }^{* *} \mathrm{P}<0,01 ;{ }^{* * *} \mathrm{P}<0,001$. 
forte diminution (65\%) de leur concentration, au cours du transit caeco-colique, la valeur correspondante pour le lot témoin étant de $58 \%$ environ.

Quelles que soient les conditions expérimentales utilisées, animaux pratiquant la caecotrophie ou non, la teneur en AGV dans le plasma artériel est en moyenne de $2 \mathrm{mM}$ (tabl. 2), l'acétate y contribue pour $88 \%$, le propionate $6 \%$ et le butyrate $6 \%$. Par contre, la lactacidémie présente des variations significatives ( $P<0,001)$ entre les deux lots d'animaux. La teneur en lactate dans le plasma artériel chute de $30 \%$ chez les sujets porteurs de collier depuis 4 jours.

Lorsqu'une activité bactérienne se manifeste dans le matériel digestif, les produits de déchets de son métabolisme, AGV et/ou lactate, peuvent se retrouver dans la circulation portale (tabl. 2). Dans les conditions physiologiques normales, le lactate apparaît dans le sang veineux en provenance de l'estomac et de l'intestin grêle $(P<0,01)$; cet apport est interrompu lors d'un jeûne stercoral de 4 jours et le taux de lactate dans le sang porte chute alors de $30 \%$ en moyenne. L'arrivée des AGV dans le plasma efférent se produit essentiellement au niveau du caecum et du côlon proximal. II en résulte au niveau de ces organes des différences veino-artérielles positives très fortes $(P<0,001)$, différences qui apparaissent significativement plus élevées lorsque les sujets expérimentaux sont privés d'alimentation caecotrophique ( $P<0,001)$. Selon les lots d'animaux, le plasma porte contient de 4,4 à 5,5 mM d'AGV qui se répartissent de la façon suivante : acétate $73 \%$, propionate $10 \%$ et butyrate $17 \%$.

Le tissu hépatique capte une fraction plus ou moins importante d'AGV et de lactate qui lui sont livrés par le sang porte et artériel (fig. 1). Pour le lot témoin,
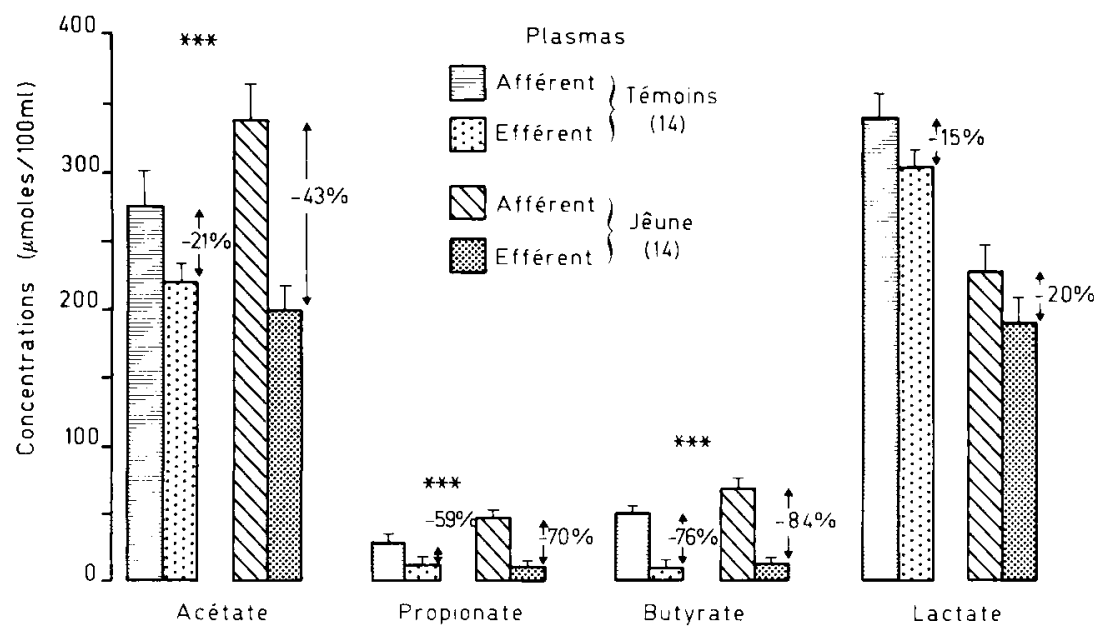

FIG. 1. - Les effets d'un jeûne stercoral de 4 jours sur les concentrations en acides gras volatils et en lactate dans les plasmas hépatiques (afférent, efférent).

- movenne $\pm \mathrm{SE}$

- ( ) : nombre d'échantillons

- Test de Student : comparaison témoins/jeûne (captation, production)

- signification : ${ }^{* *} \mathrm{P}<0,001$. 


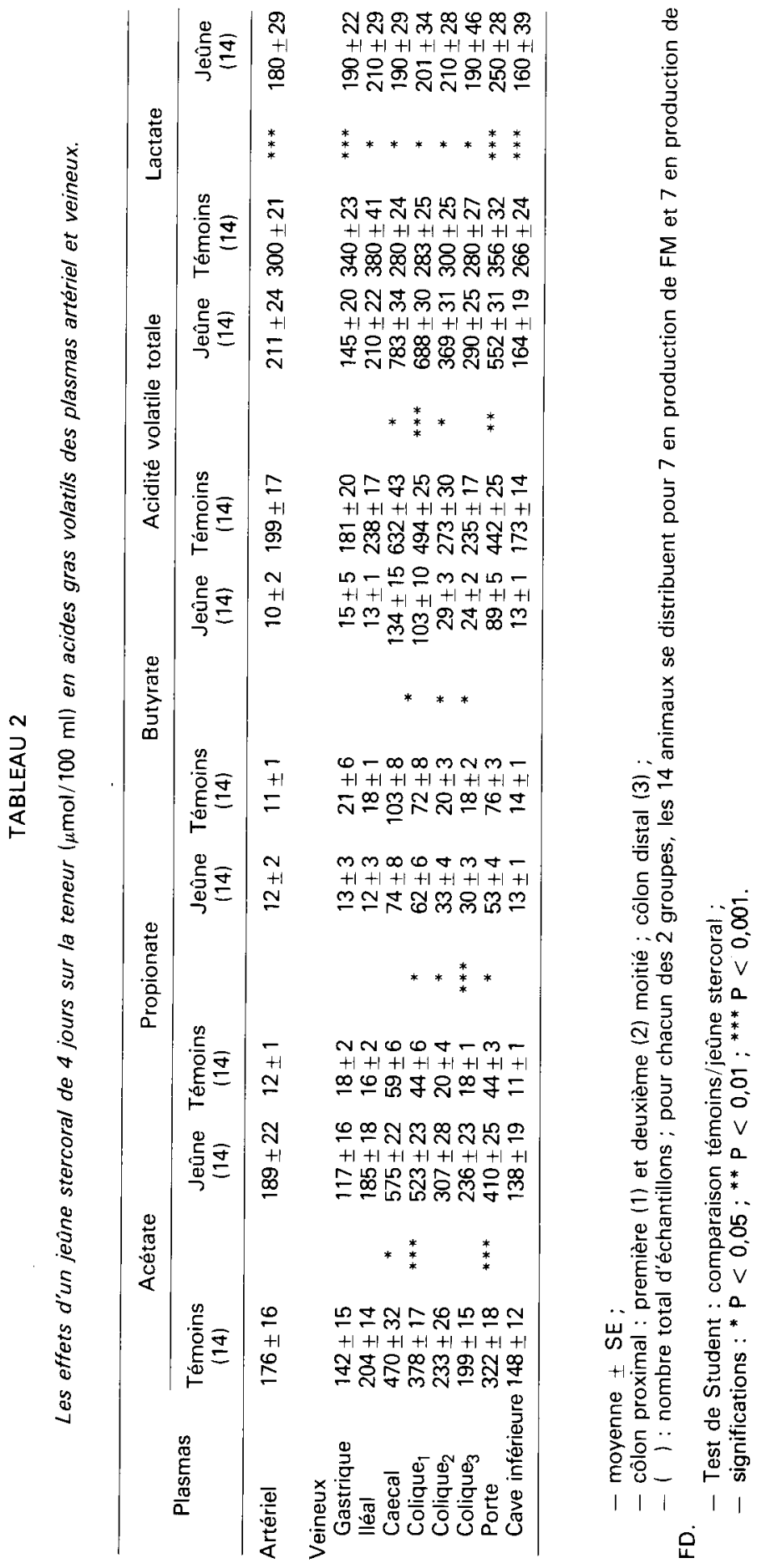


cette captation est de $15 \%$ pour le lactate, $21 \%$ pour l'acétate, $59 \%$ pour le propionate et $76 \%$ pour le butyrate. Lors du jeûne stercoral, les variations dans I'approvisionnement du foie en AGV exogènes ne s'accompagnent pas de modifications significatives de leur taux dans la veine hépatique et dans le sang artériel (tabl. 2). Dans ces conditions, l'utilisation métabolique de ces acides organiques dans cet organe est significativement augmentée de 21 à $43 \%$ pour l'acétate, de 59 à $70 \%$ pour le propionate et de 76 à $84 \%$ pour le butyrate (fig. 1 ).

Les prélèvements effectués au niveau de la veine cave inférieure (tabl. 2) font ressortir que l'acétate et le lactate sont normalement utilisés par les tissus périphériques, l'utilisation de l'acétate devenant plus intense $(P<0,001)$ lors du jeûne stercoral.

\section{Discussion.}

La privation d'alimentation stercorale, en empêchant le renouvellement de la microflore au niveau gastrique, entraîne un effondrement de la population bactérienne dans les régions antérieures du tube digestif, estomac - intestin grêle, cependant que dans les régions plus postérieures, caecum - côlon, la microflore n'est pas modifiée qualitativement et quantitativement, tout au moins pendant une courte durée du jeûne stercoral (Smith, 1965 ; Coudert, 1967 ; Yoshida et al., 1968). La quasi-disparition des microorganismes dans la première partie du tube digestif détermine un arrêt des processus fermentaires dans le bol alimentaire qui y transite, ce qui explique l'effondrement du taux des métabolites d'origine microbienne: AGV et/ou lactate. Dans l'intestin grêle, ainsi que dans le gros intestin, l'activité de la microflore est principalement orientée vers la production d'AGV, la fermentation lactique étant pratiquement inexistante (Vernay, 1985). L'apport endogène continu de lactate par la bile, essentiellement, mais aussi par les sécrétions pancréatique et intestinale (Le Bars et al., 1969 ; Vernay, 1986), contribue à élever le taux de ce métabolite du duodénum au jéjunum. Cet apport endogène ne semble pas affecté par le port du collier. Le problème se pose de comprendre pourquoi la non-ingestion des caecotrophes entraîne une hausse de l'acidité volatile dans la deuxième partie du tube digestif, hausse qui a déjà été signalée par plusieurs chercheurs (Le Bars et al., 1971 ; Vernay et Raynaud, 1975).

Le port du collier ne modifie pas de façon significative l'ingestion d'aliment, ce qui rejoint les observations de différents auteurs (Kulwich, Pearson et Lankenau, 1954 ; Proto, 1973 ; Hörnicke et al., 1976 ; Stephens, 1977 ; Catala et Bonnafous, 1978). L'accroissement de l'activité microbienne dans les régions postérieures du tractus digestif n'est donc pas le fait d'un apport supplémentaire en luzerne et avoine. Cependant, en abolissant les processus fermentaires qui se déroulent normalement dans l'estomac et l'intestin grêle et, en empêchant la décomposition de substrats d'origine endogène (mucoprotéines, enzymes, cellules desquamées, etc...) le jeûne stercoral permet l'arrivée d'une plus grande quantité de substrats fermentescibles dans le caecum ; on conçoit dès lors que les fermentations puissent s'en trouver exaltées. II convient de préciser aussi que la noningestion de caecotrophes ne modifie pas significativement le transit intestinal 
(Fioramonti et Ruckebusch, 1974) ; un ralentissement aurait augmenté le temps de séjour du bol alimentaire dans le gros intestin et favorisé ainsi l'action des microorganismes.

Dans les conditions physiologiques normales, les produits de déchets du métabolisme bactérien, $\mathrm{AGV}$ et lactate, se retrouvent dans la circulation portale. Pour les animaux témoins, les teneurs en $A G V$ que nous enregistrons à ce niveau sont du même ordre de grandeur que celles rapportées par différents auteurs, lorsque les sujets sont nourris ad libitum de luzerne et d'avoine (Cools et Jeuniaux, 1961 ; Beauville, Raynaud et Vernay, 1974 ; Bonnafous et Raynaud, 1978). Chez le lapin, de même que chez les autres monogastriques : porc (Watanabe et Murai, 1968), rat (Mottaz et Worbe, 1972 ; Rémésy et Demigné, 1976) et l'homme (Dawson, Holdsworth et Webb, 1964), ce sont les régions terminales du tractus digestif qui livrent à la circulation mésentérique une forte potentialité énergétique et plastique, sous la forme des AGV (Henning et Hird, 1972b ; Beauville, Raynaud et Vernay, 1974 ; McMillan, Edwards et Parker, 1975 ; Parker, 1976). Cependant, il n'est pas exclu que I'utilisation de ces métabolites contribue, dans un premier temps, aux besoins énergétiques des parois digestives elles-mêmes (Marty et Vernay, 1984 ; Marty, Vernay et Abravanel, 1985 ; Vernay, 1985).

Le tissu hépatique capte une fraction plus ou moins importante des AGV qui lui sont livrés par le sang porte et artériel. Nous rappelons que cette captation est de 21 à $43 \%$ pour l'acétate, de 59 à $70 \%$ pour le propionate et de 76 à $84 \%$ pour le butyrate. Si l'étude des différences artério-veineuses donne des résultats intéressants sur les flux comparés de métabolites dans une situation physiologique donnée, cette approche n'est plus suffisante lorsqu'on compare des situations pour lesquelles les débits sanguins sont très différents, par exemple : animaux normalement alimentés ou soumis au jeûne alimentaire (Bergman et Wolff, 1971 ; Rémésy et Demigné, 1983). Pour les lapins porteurs de collier, nous n'avons pas de données bibliographiques, mais il a été montré, chez le ruminant (Dobson et Phillipson, 1956 ; Bensadoun, Paladines et Reid, 1962 ; Sellers, 1965). le chien (Kvietys et Granger, 1981) et le rat (Demigné et Rémésy, 1985) qu'une hausse du taux des AGV dans le contenu digestif et dans le sang veineux correspondant accroît le débit sanguin. Peut-être en est-il de même pour le lapin ? Dans cette éventualité, l'évaluation que nous faisons de la captation hépatique est sous-estimée chez les sujets porteurs de collier. Chez le ruminant, elle est de $4 \%$ seulement pour l'acétate et de 80 à $100 \%$ pour le propionate, ainsi que pour le butyrate (Annison, Hill et Lewis, 1957 ; Cook et Miller, 1965 ; Bergman et Wolff, 1971 ; Baird, Symonds et Ash, 1975). Chez le rat, le prélèvement hépatique concerne $50 \%$ de l'acétate qui y transite et 85 à $95 \%$ pour les deux autres AGV (Rémésy, Demigné et Chartier, 1980). Pour ce qui concerne le butyrate, il convient de rappeler que dans le sang porte du lapin, cet acide organique représente, en moyenne, $17 \%$ de l'acidité volatile totale (Henning et Hird, 1972b ; Beauville, Raynaud et Vernay, 1974 ; McMillan, Edwards et Parker, 1975) alors que chez le ruminant (McClymont, 1951 ; Cook et Miller, 1965 ; Bergman et Wolff, 1971) et le porc (Friend, Nicholson et Cunningham, 1964) il n'est présent que pour 1,5 à $5 \%$ et de 5 à $11 \%$ chez le rat (Rémésy et Demigné, 1976). Donc, la capacité d'utilisa- 
tion du butyrate par le tissu hépatique du lapin est nettement plus importante que pour les autres espèces (Vernay et Marty, 1984).

L'absence de variations du taux des AGV dans les plasmas hépatique et artériel des animaux soumis au jeûne stercoral, montre qu'il existe dans cet organe une régulation du devenir de ces métabolites, régulation qui a été également envisagée chez le rat (Buckley et Williamson, 1977; Rémésy, Demigné et Chartier, 1980). Il est maintenant reconnu que, chez le lapin, les variations du taux de l'aldostérone plasmatique influencent l'absorption caeco-colique de l'eau, des ions et des AGV d'une part et le métabolisme cellulaire de l'entérocyte et de l'hépatocyte d'autre part (Clauss, 1984, 1985 ; Vernay et Marty, 1984 ; Vernay, Marty et Moatti, 1984). En bref, lorsque l'aldostéronémie est élevée, l'absorption et le métabolisme des AGV sont exaltés (Vernay, 1985). En référence à ces données bibliographiques, deux mécanismes sont susceptibles d'entraîner une plus forte activité des glandes surrénales pour les lapins porteurs de collier : le stress et/ou le déséquilibre de la balance sodée.

La lactacidémie chez le lapin est normalement élevée, de 3,9 à 8,8 mM, avec des variations physiologiques liées au régime alimentaire et à la pratique de la caecotrophie (Alexander et Chowdhury, 1958 ; Griffiths et Davies, 1963 ; Hörnicke et Mackiewicz, 1976 ; Stangassinger, Wallenberg et Giesecke, 1982 ; Vernay, 1985). Chez le lapin, de même que chez le rat (Rémésy, Demigné et Aufrère, 1977), les fermentations microbiennes au niveau de l'estomac ainsi que les processus d'absorption et de métabolisme intestinal (Marty et Carles, 1973 ; Vernay, 1985) conduisent à l'enrichissement du sang porte en lactate. De nos résultats, il ressort que le jeûne stercoral entraîne une chute $(30 \%)$ du taux de lactate dans la circulation porte et artérielle, ce qui confirme que dans les conditions physiologiques normales, une partie du lactate plasmatique trouve son origine dans le lactate produit par les bactéries contenues dans les caecotrophes. On admet généralement que ce métabolite est retenu par le foie en quantité plus ou moins grande et utilisé de façons diverses (oxydation, néoglucogenèse et/ou lipogenèse) contrairement à la plupart des espèces : ruminants (Baird et al., 1977), rat (Phillips et Hird, 1977 ; Rémésy, Demigné et Chartier, 1980), cobaye et poulet (Sarkar, 1971), le rôle glucoformateur du lactate est peu important chez le lapin (Drury et Wick, 1965). Nous avons été amenés à penser (Vernay, 1985, 1986) en accord avec Le Bars et al., (1969) qu'une partie du lactate qui parvient au foie est éliminée par la bile.

Ajoutons que, chez le lapin (Jones et Parker, 1977, 1981), de même que chez les ruminants (Bergman et Wolff, 1971; Hood, Thompson et Allen, 1972 ; Pethick et al., 1981) ou bien même le rat (Houghton et Ruderman, 1971 ; Rémésy et Demigné, 1976), l'acétate et le lactate sont bien utilisés par les tissus extrahépatiques, cette utilisation étant plus intense lorsque l'animal est soumis au jeûne stercoral. Par ailleurs, des expériences réalisées in vivo et in vitro ont permis de démontrer que, chez le lapin, le tissu adipeux privilégie l'acétate par rapport au glucose dans la synthèse des acides gras libres (Saggerson, 1974 ; Smith, 1975 ; Vézinhet et Nouguès, 1977).

La privation du complément alimentaire que constitue l'ingestion des caecotrophes chez le lapin est, au moins partiellement, compensée grâce à la stimulation de la production et l'absorption caeco-colique des AGV d'une part et de leur 
utilisation par les tissus hépatique et périphérique d'autre part, ce qui va dans le sens des résultats obtenus par Stephens (1977) et par Fraga et De Blas (1977). Ces auteurs notent que le jeûne stercoral n'entraîne pas de variations significatives du CUDa des constituants membranaires et du CUD de l'énergie ingérée.

Reçu en décembre 1985. Accepté en juillet 1986.

\section{Références}

ALEXANDER F., CHOWDHURY A. K., 1958. Digestion in the rabbit's stomach. Brit. J. Nutr., 12, $65-73$.

ANNISON E. F., HILL K. J., LEWIS D., 1957. Studies on the portal blood of sheep. 2 - Absorption of volatile fatty acids from the rumen of the sheep. Biochem. J., 66, 592-599.

BAIRD G. D., REID I. M., LOMAX M. A., SYMONDS C. J., ROBERTS C. J., MATHER D., 1977. Hepatic gluconeogenesis and fat metabolism in fed and fasted lactating dairy cows in vivo. Proc. Nutr. Soc. Biol., 36, 40 A.

BAIRD G. D., SYMONDS H. W., ASH R. W., 1975. Some observations on metabolite production and utilization in vivo by the gut and liver of adult dairy cows. J. agric. Sci., 85, 281-296.

BATTAGLINI M. B., 1968. Importanza della coprofagia nel coniglio domestico in rapporto alla utilizzazione di alcuni principi nutritivi. Rev. Zootech. Agric. Vet., 6. 21-37.

BEAUVILLE M., RAYNAUD P., VERNAY M., 1974. Concentration des acides gras volatils chez le lapin. Ann. Rech. vét., 5, 407-411.

BENSADOUN A., PALADINES O. L., REID J. T., 1962. Effect of level of intake and physical form of the diet on plasma glucose concentration and volatile fatty acid absorption in ruminants. $J$. Dairy Sci., 45, 1203-1210.

BERGMAN E. N., WOLFF J. E., 1971. Metabolism of volatile fatty acids by liver and portal-drained viscera in sheep. Amer. J. Physiol., 221, 586-592.

BITO L. Z., EAKINS K. E., 1969. The effect of general plasma of normal rabbits. J. Pharmaco/. exper. Therapeut., 169, 277-286.

BONNAFOUS R., RAYNAUD P., 1978. Etude sur la concentration des AGV du matériel et des AGV et corps cétoniques plasmatiques au niveau des veines du gros intestin chez le lapin domestique. $2^{e}$ Journ. Rech. Cunicole, Toulouse, Commun. $n^{\circ} 5$, ASFC Paris ed.

BUCKLEY B. M., WILLIAMSON D. H., 1977. Origin of blood acetate in the rat. Biochem. J., 166, $539-545$.

CATALA J., BONNAFOUS R., 1978. Modifications quantitatives de la microflore, de l'excrétion fécale et du transit intestinal chez le lapin, après ligature du canal pancréatique. $2^{e}$ Journ. Rech. Cunicole, Toulouse. Commun. $n^{\circ} 4$, ASFC Paris ed.

CLAUSS W., 1984. Circadian rhythms in $\mathrm{Na}^{+}$transport, 273-283. In SKADHAUGE E., HEINTZE K., Intestinal absorption and secretion. Lancaster MTP Press.

CLAUSS W., 1985. Segmental action of aldosterone on water and electrolyte transport across rabbit colon in vivo. Comp. Biochem. Physiol., 81 A, 873-877.

COOK R. M., MILLER L. D., 1965. Utilization of volatile fatty acids in ruminants. I - Removal of them from portal blood by the liver. J. Dairy Sci., 48, 1339-1345.

COOLS A., JEUNIAUX C., 1961. Fermentation de la cellulose et absorption des acides gras volatils au niveau du caecum de lapin. Arch. int. Physiol. Biochim., Belg., 69, 1-8.

COUDERT F., 1967. Recherches sur l'activité des sucs digestifs sur la microflore de l'estomac et de l'intestin du lapin. Th. $3^{\text {e }}$ cycle, Toulouse III.

DAWSON A. M., HOLDSWORTH C. D., WEBB J., 1964. Absorption of short chain fatty acids in man. Proc. Soc. exp. Biol. Med., 177, 97-100.

DEMIGNÉ C., REMÉSY C., 1985. Stimulation of absorption of volatile fatty acids and minerals in the cecum of rats adapted to a very high fiber diet. J. Nutr., 115, 53-60.

DESBALS P., 1963. Effets de la surrénalectomie et du pentobarbital sur la circulation des g/ucides chez le lapin. Th. $3^{\text {e }}$ cycle, Toulouse III. 
DOBSON A., PHILLIPSON A. T., 1956. Influence of the contents of the rumen and of adrenaline upon its blood supply. J. Physiol., 133, 76-77.

DRURY D. R., WICK A. N., 1965. Chemistry and metabolism of $L(+)$ and $D(-)$ lactic acids. Ann. N. Y. Acad. Sci, 119, 1061-1069.

ELSDEN S. R., HITCHCOCK M. W. S., MARSHALL R. A., PHILLIPSON A. T., 1946. Volatile fatty acids in the digesta of ruminant and other animals. J. exp. Biol., 22, 191-202.

FIORAMONTI J., BUENO L., CANDAU M., 1978. Motricité digestive et glucides membranaires chez le lapin. $2^{\theta}$ Journ. Rech. Cunicole, Toulouse, Commun. $n^{\circ} 2$, ASFC Paris ed.

FIORAMONTI J., RUCKEBUSCH Y., 1974. La motricité caecale chez le lapin. II - Variations d'origine alimentaire. Ann. Rech. vét., 2, 201-212.

FRAGA M. J., DE BLAS C., 1977. Influencia de la coprophagia sobre la utilizacion digestive de los alimentos por el conejo. Produz. anim., 8, 43-47.

FRIEND D. W., NICHOLSON J. W. G., CUNNINGHAM H. M., 1964. Volatile fatty acids and lactic acid content of pig blood. Canad. J. anim. Sci., 44, 303-308.

GIESECKE D., FABRITIUS A., WALLENBERG P. V., 1981. A quantitative study on the metabolism of $\mathrm{D}(-)$ lactic acid in the rat and the rabbit. Comp. Biochem. Physiol., 69 B, 85-89.

GRIFFITHS M., DAVIES D., 1963. The role of the soft pellets in the production of lactic acid in the rabbit stomach. J. Nutr., 80, 171-180.

GReENWAY G. V., STARK R. D., 1971. Hepatic vascular bed. Physiol. Rev., 51, 23-65.

HENNING S. J., HIRD F. J. R., 1972a. Diurnal variations in the concentration of volatile fatty acids in the alimentary tract of wild rabbits. Brit. J. Nutr., 27, 57-64.

HENNING S. J., HIRD F. J. R., 1972b. Ketogenesis from butyrate and acetate by the caecum and the colon of rabbits. Biochem. J., 130, 785-790.

HOHORST H. J., 1965. L-(+)-Lactate, determination with lactic dehydrogenase and DPN, 266277. In BERGMEYER H. U., Methods of enzymatic analysis. Acad. Press, London et NewYork.

HOOD R. L., THOMPSON E. H., ALLEN C. E., 1972. The role of acetate, propionate and glucose as substrates for lipogenesis in bovine tissues. Int. J. Biochem., 3, 598-606.

HÖRNICKE H., BJÖRNHAG G., 1980. Coprophagie and related strategies for digesta utilization. 708-730. In RUCKEBUSCH Y., THIVEND P., Digestive physiology and metabolism in ruminants, MTP Press Ltd, Lancaster, England.

HÖRNICKE H., MACKIEWICZ A., 1976. Production d'amylase, décomposition de l'amidon et formation des acides $\mathrm{D}$ et $\mathrm{L}$ lactiques par les caecotrophes. $7^{\text {er }}$ Congr. int. cunicole, Dijon Commun. $n^{\circ} 53$, ASFC Paris ed.

HÖRNICKE H. DIETZEL F., AIGNER E., SCHULTE R., 1976. Alimentary behaviour in rabbits under different influences. Fer Congr. int. cunicole, Dijon Commun. $n^{\circ} 13$, ASFC Paris ed.

HOUGHTON C. R. S., RUDERMAN N. B., 1971. Aceto-acetate as a fuel for perfused rat skeletal muscle. Biochem. J., 121, 15-16.

HUANG T. C., ULRICH E. H., McCAY C. M., 1954. Antibiotics, growth, food utilization and use of chronic oxide in studies with rabbit. J. Nutr., 54, 621-630.

JONES C. S., PARKER D. S., 1977. Arteriovenous differences studies on the lactating rabbit mammary gland. Proc. Nutr. Soc., 26, 2 A.

JONES C. S., PARKER D. S., 1981. The metabolism of glucose, acetate and palmitate in the lactating rabbit. Comp. Biochem. Physiol., 69 B. 837-842.

KAMETAKA M., 1967. Studies on the digestion in the rabbit. Part XI - Some physico-chemical properties of digesta in the small intestinal tract of the rabbit fed on a pellet diet. Agric. biol. Chem. Jap., 31, 616-624.

KULWICH R., PEARSON P. B., LANKENAU A. H., 1954. Effect of coprophagy upon the $\mathrm{S}^{35}$ uptake by rabbits after ingestion of labelled sodium sulfate. Arch. Biochem. Biophys., 50, 180-187.

KULWICH R., STRUGLIA L., PEARSON P. B., 1953. The effect of coprophagy on the excretion of $B$ vitamins by the rabbit. J. Nutr., 49, 639-645.

KVIETYS P. R., GRANGER D. N., 1981. Effect of volatile fatty acids on blood flow and oxygen uptake by the dog colon. Gastroenterology, 80, 962-969.

LE BARS H., GUÉMON L., DEMAUX G., 1971. Production d'acides gras volatils dans le caecum du lapin. Ann. Biol. anim. Biochim. Biophys., 11, 301-302. 
LE BARS H., MAHBOUB S., DEMAUX G., GUÉMON L., 1969. Existence d'un cycle entérohépatique de l'acide lactique chez le lapin. Journ. Etudes sur le lapin, Alfort, 1-12.

MARTY J., CARLES J., 1973, Absorption des acides carboxyliques par la paroi caecale du lapin Ann. Biol. anim. Biochim. Biophys., 13, 453-464.

MARTY J., VERNAY M., 1984. Absorption and metabolism of the volatile fatty acids in the rabbit hind-gut. Brit. J. Nutr., 51, 265-277.

MARTY J., VERNAY M., ABRAVANEL G., 1985. Acetate absorption and metabolism in the rabbit hind-gut. Gut, 26, 562-569.

MCCLYMONT G. L., 1951. Identification of the volatile fatty acid in the peripheral blood and rumen of cattle and the blood of other species. Austr. J. agric. Res., 2, 92-103.

MCMILLAN R. T., EDWARDS N. A., PARKER D. S., 1975. Observations on the volatile fatty acids present in the hind-gut and in the blood of the domestic rabbit. Proc. Nutr. Soc., 34, $21 \mathrm{~A}$. $22 \mathrm{~A}$.

MEGARD J. P., 1970. Aspect alimentaire de la caecotrophie chez le lapin. Th. Doct. vét., Alfort.

MOTTAZ P., WORBE J. F., 1972. Taux des AGV dans la veine caecale du rat. Influence du contenu caecal. J. Physiol., 65, 275 A.

PARKER D. S., 1976. The measurement of production rates of volatile fatty acids in the caecum of the conscious rabbit. Brit. J. Nutr., 36, 61-78.

PETHICK D. W., LINDSAY D. B., BARKER P. J., NORTHROP A. J., 1981. Acetate supply and utilization by the tissues of sheep in vivo. Brit. $J$. Nutr., 46, 97-110.

PHILLIPS J. W., HIRD F. J. R., 1977. Gluconeogenesis in vertebrate livers. Comp. Biochem. Physiol., 57 B, 127-131.

PROTO V., 1973. In cieco, in rapporto alla coprophagia nel coniglio. Produz. anim., 5, 311-316.

PROTO V., 1980. Alimentazione del coniglio da carne. Coniglicoltura, 17, 17-32.

RÉMÉSY C., 1973. Contribution à l'étude de la production et du métabolisme des acides gras volatils chez le rat. Thèse de $3^{\mathrm{e}}$ cycle, Clermont II.

RÉMÉSY C., DEMIGNÉ C., 1974. Determination of volatile fatty acids in plasma after ethanolic extraction. Biochem. J., 141, 85-91.

RÉMÉSY C., DEMIGNÉ C., 1976. Partition and absorption of volatile fatty acids in the alimentary canal of the rat. Ann. Rech. vét., 7, 39-55.

RÉMÉSY C., DEMIGNE C., 1982. Impaired lactate utilization in livers of rats fed high protein diets. J. Nutr., 112, 60-69.

RÉMÉSY C., DEMIGNÉ C., 1983. Changes in availability of glucogenic and ketogenic substrates and liver metabolism in fed or starved rats. Ann. Nutr. Métabol., 27, 71-77.

RÉMÉSY C., DEMIGNÉ C., AUFRÉRE J., 1977. Etude in vivo chez le rat, de la production et de I'utilisation de l'acide lactique. Rôle du tube digestif. Ann. Biol. anim. Biochim. Biophys., 17, 607-613.

RÉMÉSY C., DEMIGNÉ C., CHARTIER F., 1980. Origin and utilization of volatile fatty acids in the rat. Reprod. Nutr. Dévelop., 20, 1339-1349.

SAGGERSON E. D., 1974. Lipogenesis in rabbit isolated fat-cells. Biochem. J., 142, 477-482.

SARKAR N. K., 1971. Gluconeogenesis and the factors that control the process in chickens. Life Sci., 10, 293-300.

SELLERS A. F., 1965. In R. W. DOUGHERTY, Physiology of digestion in the ruminant, 171-184 Butterworths London.

SMITH H. W., 1965. The development of the flora of the alimentary tract in young animals. $J$. Pathol. Bacteriol, 90, 495-513.

SMITH S., 1975. Lipogenesis in rabbit adipose tissue. J. Lipid Res., 16, 324-331.

SPREADBURY D., 1978. A study of the protein and amino acid requirements of the growing New Zealand white rabbit with emphasis on lysine and the sulfur containing aminoacids. Brit. J. Nutr., 39, 601-613.

STANGASSINGER M., WALLENBERG P., GIESECKE D., 1982. Caecotrophy-dependent changes in the metabolic fate of D (-) lactic acid in rabbits. Arch. int. Physiol. Biochim., 90, 347-354.

STEPHENS A. G., 1977. Digestibility and coprophagy in the growing rabbit. Proc. Nutr. Soc., 26, 4 A.

VERNAY M., 1985. L'absorption et le devenir des acides gras volatils digestifs chez le lapin, en relation avec la dualité de l'émission fécale. Th. Doct. Etat, Univ. Paul Sabatier, Toulouse III. 
VERNAY M., 1986. Présence de L lactate dans les sécrétions digestives du lapin. Ann. Zootech. (sous presse).

VERNAY M., MARTY J., 1984. Absorption and metabolism of butyric acid in rabbit hind-gut. Comp. Biochem. Physiol., 77 A, 89-96.

VERNAY M., MARTY J., MOATTI J. P., 1984. Absorption of electrolytes and volatile fatty acids in the hind-gut of the rabbit. Circadian rhythm of hind-gut electrolytes and plasma aldosterone. Brit. J. Nutr., 52, 419-428.

VERNAY M., RAYNAUD P., 1975. Répartition des acides gras volatils dans le tube digestif du lapin domestique. Ann. Rech. vét., 6, 357-377.

VÉZINHET A., NOUGUĖS J., 1977. Postnatal evolution of lipogenesis in adipose tissue and liver of lamb and rabbit. Ann. Biol. anim. Biochim. Biophys., 17, 851-863.

WATANABE Y., MURAI H., 1968. The production of volatile fatty acid, lactic acid and glucose and arterio-venous differences of volatile fatty acid in sections of the alimentary tract of pig. J. Fac. Agric., Shinshu Univ. Jap., 5, 29-36.

YOSHIDA T., PLEASANTS J. R., REDDY B. S., WOSTMANN B. S., 1968. Efficiency of digestion in germ-free and conventional rabbits. Brit. J. Nutr., 22, 723-737. 